\title{
Opportunities, and Challenges of Latrine Utilization among Rural Communities of Awabel District, Northwest Ethiopia, 2014
}

\author{
Molla Gedefaw1 ${ }^{*}$, Yibeltal Amsalu1, Molalign Tarekegn1, Worku Awoke² \\ ${ }^{1}$ GAMBY College of Medical Sciences, Bahir Dar, Ethiopia \\ ${ }^{2}$ College of Medicine and Health Sciences, Bahir Dar University, Bahir Dar, Ethiopia \\ Email: yebaeamsa@gmail.com, ${ }^{*}$ mollagedefaw@yahoo.com, tamolalign@gmail.com,workuawo@yahoo.com
}

Received 6 February 2015; accepted 11 April 2015; published 15 April 2015

Copyright (C) 2015 by authors and Scientific Research Publishing Inc.

This work is licensed under the Creative Commons Attribution International License (CC BY).

http://creativecommons.org/licenses/by/4.0/

(c) () Open Access

\begin{abstract}
Ethiopia is working hard to increase access to improved sanitation to its rapidly growing population; however, its proper utilization is questioned. Update on its utilization is required. The objective of the study was to assess latrine utilization and associated factors among rural communities in Awabel District, Northwest Ethiopia, 2014. Community based cross-sectional study was conducted on 575 households. Participants were selected through multi-stage sampling technique. Pre-tested interviewer administered structured questioner and observational checklist was used to collect data. The collected data were coded and entered using Epi-Info 3.5.3 and analysis was done using SPSS version 20. Bivariable and multivariable logistic regressions were used to identify the associated factors for proper utilization of latrine facilities. Possible association and statistical significance were measured using odds ratio at $95 \%$ confidence interval. Proper utilization of latrine was practiced by about 297 (52\%) of households. Utilization was significantly associated with presence of school children in the households [AOR: 1.824, 95\%CI (1.189 - 2.799)], and latrine which did not need maintenance [AOR: 4.294, 95\%CI (2.625 - 7.025)], households who were advised to construct latrine by health professionals [AOR: 4.194, 95\%CI: (1.674 - 12.358)] and self initiation to construct latrine [AOR: $7.461,95 \% \mathrm{CI}$ : $(1.850-30.084)]$. The most outstanding findings of this study were the following: latrine utilization will be sustainable when the toilet is constructed following proper household based health education, when the toilet is well constructed, and when there is supportive supervision. This implies that health extension workers do have a pivotal role for increasing toilet construction as well as its proper utilization. The current level of proper latrine utilization seems fairly good for rural communities in the district. However, there is a lot to do to make it part of their culture. Its sustainability seems to be achievable via health extension package. Therefore, motivating health extension workers is recommended.
\end{abstract}

${ }^{*}$ Corresponding author.

How to cite this paper: Gedefaw, M., Amsalu, Y., Tarekegn, M. and Awoke, W. (2015) Opportunities, and Challenges of Latrine Utilization among Rural Communities of Awabel District, Northwest Ethiopia, 2014. Open Journal of Epidemiology, 5, 98-106. http://dx.doi.org/10.4236/ojepi.2015.52013 


\section{Keywords}

\section{Opportunities, Challenges, Toilet, Rural, Communities}

\section{Introduction}

Improved households sanitation has been considered for long as a cost effective intervention to reduce child and adulthood morbidity and mortality [1]. Studies estimated that proper utilization of pit latrines could reduce diarrhea by more than $36 \%$, cholera by $66 \%$, and worm infestations by between $12 \%$ and $86 \%$ [2].

World nations have been working for the past two decades to double the number of people who have access to improved sanitation by 2015 [3]. Though off the track, substantial progress was achieved in increasing the number of people who have access to improved sanitation from 49\% in 1990 to $64 \%$ in 2011 [4]. Ethiopia has also been working hard to achieve 100 percent coverage of latrine facilities [5].

However, possession of improved latrine per se will not halt the transmission of faeco-orally transmitted diseases. For this to have an impact on health, people have to use latrines and hand washing facilities effectively [6]. There are very few recent studies on toilet utilization especially among rural communities in Ethiopia. Anteneh and Kumie (2010) published an article which stated that the prevalence of toilet utilization was $61 \%$. This study was conducted in an almost similar setup, but it was conducted more than five years go. Therefore, report on the current state of affaires regarding toilet utilization—an important method for the control of diseases of poverty including diarrhea, trachoma, and intestinal paretic diseases-is needed.

The current study was aimed at determining the level of latrine utilization and its associated factors among households in Awabel District where the latrine coverage was reported to reach nearly 100\% [7]. The finding of the study will help local decision makers knowing the current status of latrine utilization, and what should be done in the future.

\section{Methods}

\subsection{Study Design}

Community based cross-sectional study was carried out in rural community of Awabel District, Northwest Ethiopia from June to July 2014. The district is located about $256 \mathrm{Km}$ from Addis Ababa (the capital city of Ethiopia) and $307 \mathrm{Km}$ from Bahir Dar (the capital city of Amhara regional State). It has an estimated population of about 131,691. It has 3 urban and 28 rural kebeles (the lowest administrative level in Ethiopia).

This study was planned to be done on 575 households. This sample size was calculated by using the single population proportion formula. The assumptions made were level of latrine utilization (34.7\%) [8]; margin of error (5\%), 95\% confidence interval, design effect (1.5) and 10\% contingency for possible non-response.

Households were selected using multi-stage sampling technique. First Kebeles were stratified based on distance from district health office where the main district health center is located. Kebeles were labeled as "far", "moderate" and "near." Then, kebels were selected using lottery method proportionally. Finally, the desired number of households were taken from each Kebele using systematic random sampling method.

The dependent variable was proper latrine utilization. Independent variables were: Socio-demographic factors: family size, educational status of the mother and father, household income, household with primary or secondary educational level children, Behavioral factors: perceived reason for latrine construction and frequency of supportive supervision, Environmental factors: years of latrine construction, latrine functionality, status of latrine, availability latrine super structure, distance from the local health facilities, distance between latrine and home, availability of hand washing facilities and accessibility to kebeles.

\subsection{Data Collection and Analysis}

Data was collected through pretested structured interviewer administered questionnaire and observational checklist. They were adopted from other similar studies [8]-[13]. It was translated into the local language and back to English again to assure consistency. Data was collected and supervised by trained professional (Diploma 
nurses and environmental health professionals). Data was checked for completeness on daily basis. It was coded, entered and cleaned using Epi-Info version 3.5 and exported to SPSS version 20 for further analysis. Univariable, bivariable and multivariable analysis were done to express the result. Possible associations were measured using Odds ratio at $95 \% \mathrm{CI}$.

\subsection{Operational Definitions}

1) Proper latrine utilization: in the current study household were considered as they were utilizing latrine properly if the compound was free of free of observable feces and has functional latrine during the study, having no observable feces around squat hole and the footpath to it was not covered by grass.

2) Functional latrine: latrine that can provide service at the time of data collection even if it requires maintenance.

3) Latrine with maintenance: latrine with damaged slab or super structure.

4) Near: households located a distance of 10 kilometers or less from the District Health Office.

5) Moderate: households located a distance of 10 - 20 kilometers from the District Health Office.

6) Far: households located a distance of over 20 kilometers from the District Health office.

\section{Ethical Considerations}

Ethical clearance was obtained from ethical review committee of GAMBY College of Medical Sciences. Letter of Permission was obtained from the Amhara regional health bureau of research and technology transfer core process, the District health Office as well as from each kebele administration. During data collection verbal consent was also obtained from study participants. Confidentiality of the information was assured throughout the data collection process.

\section{Results}

\subsection{Socio-Demographic and Economic Characteristics of Selected Households in Awabel District, Northwest Ethiopia, 2014}

All of the selected households were participated in the study making the response rate $100 \%$. About fifty four percent had an annual income of less than 1124.75 USD. Sixty three percent had five or more families. About eighty percent of households had children attending primary and above. Only about $9.4 \%$ of fathers and $5.6 \%$ of mothers in the household were literate. Majority of fathers (97.4\%) and mothers (86.6\%) were farmers and housewife, respectively (Table 1 ).

\subsection{Sanitation Facility in the Selected Households of Awabel District, Northwest Ethiopia, 2014}

Pit latrine is the only latrine available. About sixty percent of them were located 6 - 10 meters away from houses. Thirty five percent of latrines need maintenance, either to the superstructure or floor. Seventy five percent of the latrines were functional during the time of data collection and ten percent had no superstructure.

About ninety one percent of latrines had no cover on the squatting hole. Five hundred thirty four (92.9\%) households had no any kind of hand washing facilities. Only 7\% of households had hand washing facility were using either soap or ash (Table 2).

\subsection{Perceived Reasons for Latrine Construction in Selected Households of Awabel District, Northwest Ethiopia, 2014}

About $80 \%$ of study participants claimed that they constructed latrines following advice from health extension workers (Figure 1).

\subsection{Perceived Reasons for Latrine Use in Selected Households of Awabel District, Northwest Ethiopia}

More than $50 \%$ of study participants claimed that always use latrines because they knew that open defecation is 
Table 1. Socio-demographic and economic characteristics of selected households in rural communities of Awabel District, northwest Ethiopia, 2014.

\begin{tabular}{|c|c|c|c|}
\hline \multirow{2}{*}{ Variable } & \multirow{2}{*}{ Values } & \multicolumn{2}{|c|}{ Frequency } \\
\hline & & Number & Percent \\
\hline \multirow[b]{2}{*}{ Family size $(\mathrm{n}=575)$} & $\geq 5$ & 365 & 63.5 \\
\hline & $<5$ & 210 & 36.5 \\
\hline \multirow{3}{*}{ Marital status $(\mathrm{n}=575)$} & Married & 498 & 86.6 \\
\hline & Widowed & 53 & 9.2 \\
\hline & Divorce & 24 & 4.2 \\
\hline \multirow{2}{*}{ Head of household $(n=575)$} & Husband & 498 & 86.6 \\
\hline & Wife & 77 & 13.4 \\
\hline \multirow{2}{*}{ Husband occupation ( $\mathrm{n}=498$ ) } & Farmer & 485 & 97.4 \\
\hline & Daily laborer & 13 & 2.6 \\
\hline \multirow{3}{*}{ Husband education $(n=498)$} & Illiterate & 261 & 45.4 \\
\hline & Able to read and write & 183 & 31.8 \\
\hline & Literate & 54 & 9.4 \\
\hline \multirow{2}{*}{ Wife occupation ( $\mathrm{n}=575)$} & House wife & 498 & 86.6 \\
\hline & Farmer & 77 & 13.4 \\
\hline \multirow{3}{*}{ Wife education $(n=575)$} & Illiterate & 347 & 60.3 \\
\hline & Able to read and write & 196 & 34.1 \\
\hline & Literate & 32 & 5.6 \\
\hline \multirow{2}{*}{ HHs with school children $(n=575)$} & Yes & 462 & 80.3 \\
\hline & No & 113 & 19.7 \\
\hline \multirow{2}{*}{ Income $(n=575)$} & $<22,495$ birr per year & 308 & 53.6 \\
\hline & $\geq 22,495$ birr per year & 267 & 46.4 \\
\hline
\end{tabular}

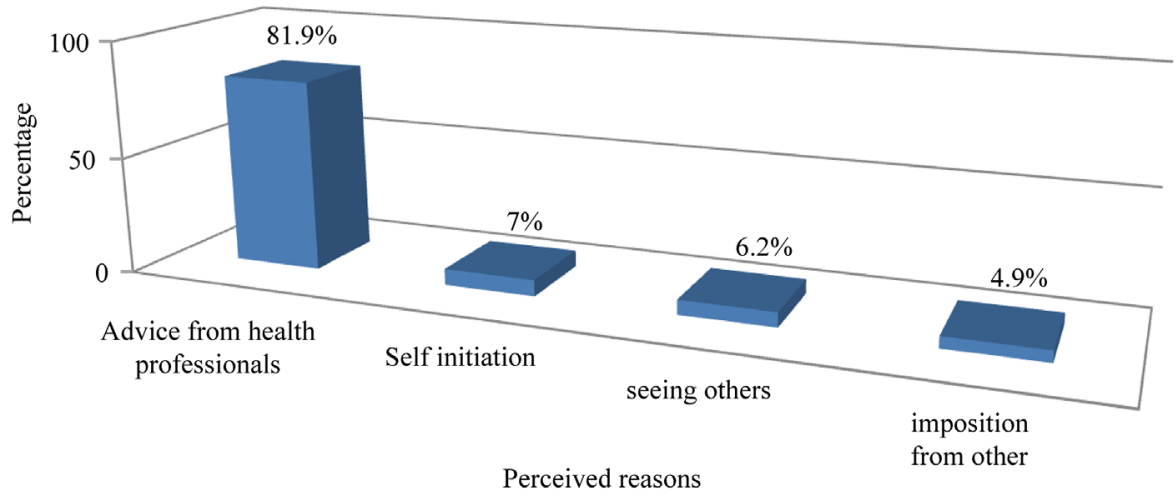

Figure 1. Perceived reasons for latrine construction, Awabel District, Northest Ethiopia, $2014(n=427)$. 
Table 2. Distribution of respondents by environmental factors among rural communities of Awabel District, Northwest Ethiopia, 2014.

\begin{tabular}{|c|c|c|c|}
\hline \multirow{2}{*}{ Variables } & \multirow{2}{*}{ Values } & \multicolumn{2}{|c|}{ Frequency } \\
\hline & & Number & Percent \\
\hline \multirow[t]{2}{*}{ Latrine year of construction $(n=575)$} & $<2$ year & 290 & 50.4 \\
\hline & $\geq 2$ year & 285 & 49.6 \\
\hline \multirow[t]{2}{*}{ Squat covered $(\mathrm{n}=575)$} & Yes & 53 & 9.2 \\
\hline & No & 522 & 90.8 \\
\hline \multirow[t]{2}{*}{ Slab covered with mud or cement $(n=575)$} & Yes & 485 & 84.3 \\
\hline & No & 90 & 13.7 \\
\hline \multirow[t]{4}{*}{ Latrine super structure $(\mathrm{n}=575)$} & Wood only & 79 & 13.7 \\
\hline & Wood \& mud & 294 & 51.1 \\
\hline & Grass & 145 & 25.2 \\
\hline & No super structure & 57 & 9.9 \\
\hline \multirow[t]{2}{*}{ Latrine condition $(\mathrm{n}=575)$} & Need maintenance & 201 & 35.0 \\
\hline & No need of maintenance & 374 & 65.0 \\
\hline \multirow[t]{4}{*}{ Maintenance part of latrine $(n=201)$} & Super structure & 44 & 21.9 \\
\hline & Slab & 36 & 17.9 \\
\hline & Roof & 26 & 12.9 \\
\hline & Mixed & 95 & 47.3 \\
\hline \multirow[t]{2}{*}{ Walking time from home to health institution (575) } & $<30$ minutes & 302 & 52.5 \\
\hline & $\geq 30$ minutes & 273 & 47.5 \\
\hline \multirow[t]{3}{*}{ Distance of Latrine from home $(\mathrm{n}=575)$} & $<6$ meters & 197 & 34.3 \\
\hline & 6 - 10 meters & 342 & 59.5 \\
\hline & $>10$ meters & 36 & 6.3 \\
\hline \multirow[t]{2}{*}{ Keble accessibility $(\mathrm{n}=575)$} & Near to average & 287 & 49.9 \\
\hline & Too far & 288 & 50.1 \\
\hline \multirow[t]{3}{*}{ Frequency of supervision $(n=575)$} & 1 - 2 times per months & 325 & 56.5 \\
\hline & 3 times and above per month & 221 & 38.5 \\
\hline & Never visited & 29 & 5.0 \\
\hline \multirow[t]{2}{*}{ Hand washing facility $(\mathrm{n}=575)$} & Yes & 41 & 7.1 \\
\hline & No & 534 & 92.9 \\
\hline \multirow[t]{2}{*}{ Availability of detergents $(n=41)$} & Nothing & 30 & 73.2 \\
\hline & Soap/ash & 11 & 26.8 \\
\hline
\end{tabular}

dangerous to health (Figure 2).

\subsection{Level of Proper Latrine Utilization in Selected Households of Awabel District, Northwest Ethiopia, 2014}

According to the current study, 297 (51.7\%) households were using latrine facility properly (Table 3). 


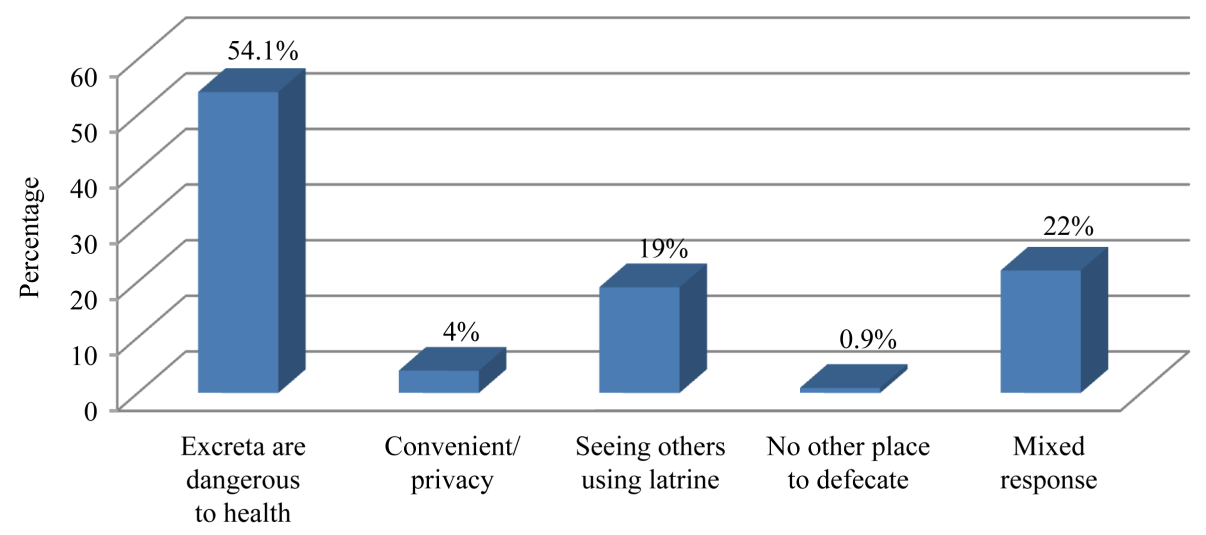

Perceived reasons

Figure 2. Reasons for using latrine by $\geq 5$ years old, Awabel District, Northwest, Ethiopia, $2014(n=427)$.

Table 3. Level of latrine utilization in selected households of Awabel District, June, 2014 (n = 575).

\begin{tabular}{|c|c|c|c|}
\hline Variables & Values & Frequency & Percent \\
\hline \multirow{2}{*}{ Functional latrine $(\mathrm{n}=575)$} & Yes & 430 & 74.8 \\
\hline & No & 145 & 25.2 \\
\hline \multirow{2}{*}{ Road covered with grass $(n=575)$} & Yes & 153 & 26.6 \\
\hline & No & 422 & 73.4 \\
\hline \multirow{2}{*}{ Fresh feces around the squat hole $(n=575)$} & Yes & 134 & 23.3 \\
\hline & No & 441 & 76.7 \\
\hline \multirow{2}{*}{ Observable feces in the compound $(\mathrm{n}=575)$} & Yes & 259 & 45.0 \\
\hline & No & 316 & 55.0 \\
\hline \multirow{2}{*}{ Proper latrine utilization $(n=575)$} & Yes & 297 & 51.7 \\
\hline & No & 278 & 48.3 \\
\hline
\end{tabular}

\subsection{Factors Associated with Proper Latrine Utilization in the Selected Households of Awabel District}

The factors that were found to have association with proper latrine utilization were the following: status of latrine, reason for latrine construction, Presence of superstructure constructed and kebeles accessibility ( $\mathrm{p}$-value $<$ $0.05)$.

Households with primary or secondary school children were 2 times more likely to utilize latrine than households with no primary or secondary school children [AOR: 1.824, 95\%CI (1.189 - 2.799)]. Moreover, latrine utilization was about 4 times more likely [AOR: 4.294 (2.625 - 7.02)] among households with latrines that do not need maintenance compared with those households whose latrine which needs maintenance.

Households who construct their latrine following advice given by health professionals were about 4 times [AOR: 4.194, 95\%CI: $(1.674-10.508)$ ] and self initiation were 7 times more likely [AOR: 7.461 (1.850 30.084) to utilize latrine than those imposed by government officials. In addition, the level of latrine utilization was three times more in households near to woreda health center [AOR, 3.359; 95\%CI (2.239 - 5.039)] than in far ones.

Those households whose latrine has superstructure were three times more likely [AOR: 3.008, 95\%CI (1.364 - 6.631)] to utilize latrine compared with households having latrine with no superstructure (Table 4). 
Table 4. Predictors of latrine utilization in Awabel District, North West Ethiopia, June 2014 (n = 575).

\begin{tabular}{|c|c|c|c|c|}
\hline \multirow{2}{*}{ Variables } & \multicolumn{2}{|c|}{ Proper latrine utilization } & \multirow{2}{*}{ Crude or $(95 \% \mathrm{CI})$} & \multirow{2}{*}{ Adjusted or $(95 \% \mathrm{CI})$} \\
\hline & Yes & No & & \\
\hline
\end{tabular}

Presence of school children

$\begin{array}{lcccc}\text { Yes } & 254 & 208 & 1.988(1.304-3.031)^{* * *} & 1.824(1.189-2.799)^{* * *} \\ \text { No } & 43 & 70 & 1.00 & 1.00 \\ \text { ome } & & & & 1.00 \\ <22,495 \text { birr per year } & 143 & 165 & 1.00 & 1.310(0.925-1.854) \\ \geq 22,495 \text { birr per year } & 145 & 113 & 1.572(1.130-2.188)^{* *}\end{array}$

Latrine super structure constructed

$\begin{array}{lcc}\text { Only with wood } & 16 & 63 \\ \text { With wood \& mud } & 173 & 121 \\ \text { By grass } & 93 & 52 \\ \text { No super structure } & 15 & 42\end{array}$

$\begin{array}{cc}0.711(0.318-1.591) & 0.684(0.277-1.689) \\ 4.003(2.124-7.54)^{* *} & 1.766(0.812-3.812) \\ 5.008(2.008-9.88)^{* *} & 3.008(1.364-6.631)^{* *} \\ 1.00 & 1.00\end{array}$

Years of latrines construction

$\begin{array}{ll}\geq 2 \text { yrs } & 168 \\ <2 \text { yrs } & 129\end{array}$

$1.021(0.678-1.537)$

$<2$ yrs

Status of latrine

\section{No need maintenance \\ Need maintenance}

Latrine squat covered

Yes

Slab covered with mud or cement

Yes

Reason of latrine construction

Health professional advice
Self initiation
Seeing other
Imposition from others

Kebele accessibility

Near to average

$4.294(2.625-7.02)^{* * *}$

1.00

$$
\begin{gathered}
3.173(1.65-6.07)^{* *} \\
1.00
\end{gathered}
$$

$1.244(0.604-2.561)$

265

73

$5.86(3.358-10.245)^{* *}$

1.00

$$
\begin{gathered}
3.859(1.609-9.245)^{*} \\
3.6665(1.087-12.35) \\
2.27(0.654-7.923) \\
1.00
\end{gathered}
$$

1.00

Too far 106
96 182
$3.416(2.425-4.81)^{* * *}$ 1.00
$1.105(0.534-2.269)$

1.00

*Significant at $\mathrm{p}<0.05,{ }^{* *}$ Significant at $\mathrm{p}<0.01,{ }^{* * *}$ Significant at $\mathrm{p}<0.001$. 


\section{Discussion}

The main objective of the current study was to assess the level of latrine utilization and associated factors among households in Awabel District, North West Ethiopia.

In the present study, about $52 \%$ of the households were using their latrine properly. The finding was in line with reports from other districts in the region. Some of these reports include the following: Hulet Ejju Enessie District (61\%) [6], and Bahir Dar Zuria District (62\%) [9]. It is higher than the finding in Hawzien District from the Northern part of Ethiopia (37.4\%) [13], and a report from Mtwara Rural District of Tanzania (40.0\%) [12]. This variation could be partly explained by the fact that the study population could have differences in socioeconomic, and other factors in addition to differences in study designs and operational definitions of the outcome of interest.

The observed level of proper latrine utilization in the studied districts of the region can be seen as a good development for households in Ethiopian rural districts because a decade or so a go most of the rural households had no any access to improved latrine facilities, and hence Ethiopia was one of the countries in the world where millions of people practiced open defecation.

In the present study, it was interesting to notice that proper latrine utilization was two times higher among households that had primary or secondary school children than those with no primary or secondary school children. This was consistent with the finding in Hulet Ejju Enessie District [6]. This could be partly explained by the fact that Ethiopian school books contain lots of information about common diseases-their causes, transmission, and prevention methods.

Hence it is a good opportunity for Ethiopia because nowadays it becomes a culture even for rural households to send their children to school. And these children could understand health messages better than their busy and uneducated mothers, and fathers. The health system should capitalize this opportunity through inter-sectoral collaboration.

The present study has also revealed that health professional's advice has a pivotal role for construction, as well as proper utilization of improved toilet facilities. This is again an important opportunity, and indicator for success of implementation of health extension package in Ethiopia that can bring about fundamental societal changes which are essential for controlling as well as elimination of especially diseases of poverty including intestinal parasites, trachoma, and diarrhea.

The present study also helped identify important challenges of communities in the study area in using improved latrine facilities. One of these factors was related to supportive supervision. According to the data of this study, proper utilization of latrine was affected by distance of the households from district health office. This result was in line with the finding from a study conducted in one of the districts in the same region [9]. This could be partly explained by the fact that households near to the district town where the district health office is located could get better supportive supervision than those households which are located far from the district health office.

Other challenges were related to construction quality of latrine facilities. Logistic regressions revealed that the level of latrine utilization were about 3 times higher (AOR: 3.135, 95\%CI: 1.781 - 5.518) among households whose latrines did not need maintenance, and has superstructure (AOR: 3.194, 95\%CI: 1.364 - 6.631) compared with those households whose latrine needed maintenance, and had no superstructure. For instance, in this study, $35 \%$ of toilet facilities need maintenance, both to the superstructure or floor. This figure was lower than the finding in Hulet Ejju Enessie (47.2\%) [6] and Bahir Dar Zuria District (56.9\%) [9].

Even though it is recommended to build latrine with a minimum distance of six meters from home (10), in the current study, only 197 (34.3\%) of latrines were located within six meters from the home. This was consistent with the finding in Bahir Dar Zuria District (32.1\%) [9]. This shows that poor quality and wrong placement of toilet construction hinders proper utilization of toilet in the rural communities in the region.

Another very critical challenge was absence of hand washing facility near to the toilet. More than $98 \%$ of toilets observed had no hand washing facility. This was in line with the finding in Bahir Dar Zuria [93.8\%] [9] but higher than the finding in Mubende District of Uganda, [82\%] [11]. These could be partly attributed to scarcity of water, and soap in concert with lack of awareness about the importance of hand washing after visiting toilet in preventing feco-oral transmission of diseases.

This study could have been more rigorous had it been supported with qualitative research approach which could have involved health extension workers, and school children as study participants. 


\section{Conclusion and Recommendation}

The current level of proper latrine utilization is fairly good for rural communities in the district. However, there is a lot to do to make it part of their culture. Its sustainability seems to be achievable via Health Extension Package, and school children. Therefore, motivating health extension workers to involve school teachers and children in their Endeavour is recommended. Challenges related to quality, and placement of toilet facility construction require attention by decision makers at district level and beyond to further increase proper toilet utilization in Ethiopia.

\section{Acknowledgements}

We would like to thank Bahir Dar University and GAMBY College of Medical Sciences for opening a joint MPH training program which increases research and training opportunities in our region. We are also very grateful for supervisors and data collectors.

\section{Conflict of Interest}

We declare that there is no conflict of interest in this research.

\section{Contribution of Authors}

YA: raised the research idea, wrote the first proposal, as well as manuscript drafts. MG, MT and WA reviewed and finalized them.

\section{References}

[1] WHO (2014) 10 Facts on Sanitation. http://www.who.int/features/factfiles/sanitation/facts/en/index1.html

[2] Ministry of Health (2005) National Hygiene and Sanitation Strategy. Addis Ababa, Ethiopia. http://www.wsp.org/sites/wsp.org/files/publications/622200751450 EthiopiaNationalHygieneAndSanitationStrategyA $\underline{\text { F.pdf }}$

[3] USIAD (2006) Achieving the Millennium Development Goals: The Contribution of Fulfilling the Unmet Need for Family Planning. http://pdf.usaid.gov/pdf_docs/PNADM175.pdf

[4] Unicef/WHO (2012) Progress on Drinking Water and Sanitation. http://www.unicef.org/media/files/JMPreport2012.pdf

[5] Federal Democratic Republic of Ethiopia, Ministry of Health (2004) Construction, Usage and Maintenance of Sanitary Latrine Extension Package. Addis Ababa. http://cnhde.ei.columbia.edu/training/documents/Sanitary_Latrine.pdf

[6] Anteneh, A. and Kumie, A. (2010) Assessment of the Impact of Latrine Utilization on Diarrhoeal Diseases in the Rural Community of Hulet Ejju Enessie Woreda, East Gojjam Zone, Amhara Region. Ethiopian Journal of Health Development, 24, 110-118. http://dx.doi.org/10.4314/ejhd.v24i2.62959

[7] The 2012/2013 Annual Report. Awabel District Health Office, Lumami.

[8] Roma, E. and Pugh, I. (2012) Toilets for Health. London School of Hygiene and Tropical Medicine.

[9] Awoke, W. and Muche, S. (2013) A Cross Sectional Study: Latrine Coverage and Associated Factors among Rural Communities in the District of Bahir Dar Zuria, Ethiopia. BMC Public Health, 13, 1-6. http://dx.doi.org/10.1186/1471-2458-13-99

[10] Abebe, A., Wale, A., Abebe, C., Sime, E., Kaba, E., Abate, M., et al. (2012) Water Supply and Sanitation in Amhara Region. Bahir Dar, Ethiopia. Learning and Communication Research Report, 54-61.

[11] Robert, M. and Kusiima, B.A. (1998) Community Use of Pit-Latrines in Mubende District. Child Health and Development Centre Ministry of Health, Health Planning Department, Uganda National Health Research Organization and UNICEF.

[12] Kema, K., Semali, I., Mkuwa, S., Kagonji, I., Temu, F., Ilako, F., et al. (2012) Factors Affecting the Utilization of Improved Ventilated Latrines among Communities in Mtwara Rural District, Tanzania. Pan African Medical Journal, 13, $1-5$.

[13] Ashebir, Y., Sharman, H.R., Alemu, K. and Kebede, G. (2013) Latrine Use among Rural Households in Northern Ethiopia: A Case Study in Hawzien District, Tigray. International Journal of Environmental Studies, 70, 629-636. http://dx.doi.org/10.1080/00207233.2013.835533 